\title{
Characterizing Work-Related Smartphone Use at Home and Private Smartphone Use at Work Using Latent Class Analysis
}

\author{
Jonas Dora, et al. [full author details at the end of the article]
}

Received: 18 April 2018 / Revised: 12 February 2019 / Accepted: 5 March 2019 / Published online: 24 May 2019

(C) The Author(s) 2019

\begin{abstract}
The smartphone can be used for two context-incongruent purposes (work-related use at home and private use at work). In order to better understand these two behaviors conceptually, we aimed to (1) identify subgroups of context-incongruent smartphone users and (2) identify differences in demographic, smartphone-related, and occupational health-related characteristics among the identified subgroups. We conducted an exploratory and datadriven latent class analysis of work-related smartphone use at home and private smartphone use at work (self-reported) in a large cross-sectional sample of Dutch fulltime employees $(n=1544)$. Our analysis revealed that most employees engage in context-incongruent smartphone use and identified four smartphone user classes. Comparisons of frequent and infrequent context-incongruent smartphone users revealed several interesting insights regarding demographic (e.g., frequent users were younger, more likely to be married or in a relationship, and less likely to work from their employer's site), smartphone-related (e.g., frequent users were more likely to be provided a smartphone by their employer, attached more importance to their work-related and private smartphone interactions, and reported higher fear of missing out), and occupational health-related (e.g., frequent users reported only slightly higher job demands, job control, and work-home interference, but at the same time lower segmentation preferences and psychological detachment) characteristics. These findings provide insight into the wide-spread occurrence of context-incongruent smartphone use and could help to develop theory on and understand the outcomes of these modern behaviors. They could also help organizations to better understand their employees' behavior, which is a crucial first step in policy development.
\end{abstract}

Keywords Work-related smartphone use · Private smartphone use · Latent class analysis · Fear of missing out · Psychological detachment · Work-home interference

The way the introduction of the smartphone has led to an enormous change in people's everyday lives, and more specifically its role in blurring the boundaries between work

Electronic supplementary material The online version of this article (https://doi.org/10.1007/s41542-01900040-6) contains supplementary material, which is available to authorized users. 
and private life, has been discussed (e.g., Demerouti et al. 2014) and empirically investigated (e.g., Derks et al. 2015) at great length. Most of the debate has focused on how the smartphone has made work more accessible in the private domain (i.e., work-related smartphone use at home). Work-related smartphone use at home can range from a quick (repetitive) check for work-related emails or reports to the reading and writing of these work-related emails or reports, answering of work-related calls, and other work-related activities that can be executed via the smartphone. Although employees themselves report that the smartphone helps them to integrate responsibilities in the work and private domain (APA Center for Organizational Excellence 2013), research has indicated that using the smartphone for work-related matters at home can impede the recovery process (e.g., Park et al. 2011) and increase work-home interference (e.g., Derks et al. 2015), which highlights the importance to study this type of smartphone use from an occupational health perspective.

Given that the smartphone has provided employees with a tool to stay connected to work outside of working hours, it stands to reason that it also allows them to stay more connected to their private life while being at work (i.e., private smartphone use at work). Similar to its counterpart, private smartphone use at work can be a short smartphone interaction such as a check for private instant messages, but also more intrusive such as the reading and answering of private messages or emails, making private calls, browsing the internet, or using various apps for entertainment purposes. However, research on this side of the coin is missing. It can be argued that private smartphone use at work can be conceptualized in a positive way (i.e., being a break from work/recovery opportunity) or negative way (i.e., being an interruption). Hence, it is important to study its potential positive and negative outcomes. Before we are able to fully understand the outcomes of a relatively new behavior, it is imperative to get a good conceptual understanding of the behavior itself. Additionally, both types of context-incongruent smartphone use can be expected to increase as organizations are giving their employees more freedom in how, when, and where to work (Demerouti et al. 2014) and 'digital natives' (younger generations that have grown up with modern technology like the smartphone; Voorveld and van der Groot 2013) make up a larger proportion of the labor force.

Conceptually, context-incongruent smartphone use can be understood in the context of boundary theory (Ashforth et al. 2000). This theory posits that individuals differ in the extent to which they separate or integrate various life domains (e.g., work and private life) due to a combination of their personal preferences as well as environmental pressures, such as organizational and peer norms. This degree of integration or separation in turn should predict how often employees engage in boundary-crossing behavior, which in turn is expected to affect related outcomes such as work-home conflict. Based on a review of the literature on (work-related) technology use during non-work time, Schlachter et al. (2017) arrive at a very similar conceptual model, in which the organizational context and individual characteristics are expected to influence the amount of work-related technology use, which in turn may lead to impaired work-life balance and recovery. Boundary theory also states that the more often a boundary-crossing behavior is performed, the more natural and automatic it will become. Given how easy it is to pick up one's smartphone and check for that work-related email one is expecting in the evening or that message by one's 
daughter after an exam during the day, it is conceivable how these behaviors could quickly be internalized. However, one limitation of the applicability of boundary theory to the study of context-incongruent smartphone use is that we do not yet know whether work-related smartphone use at home and private smartphone use at work are truly symmetrical, mirrored behaviors (i.e., are they highly correlated, are they related to similar psychological constructs?).

In summary, initial evidence suggests that work-related smartphone use at home may be associated with lower individual well-being, while private smartphone use at work has not received any attention. As a consequence, these behaviors have not been studied together, and we do not know the extent to which they share an underlying 'user profile'. Given that this field is in its infancy, it is important to study the individual and environmental context in which both types of smartphone use take place, which will aid in developing theory and in understanding their effects on potential outcomes (e.g., recovery and productivity). From a practical perspective, it is important to get a better estimate of the frequency of both types of use and to understand their context. For example, if organizations better understand why employees (excessively) engage in these behaviors, they may be able to develop policies that target these perceived needs or necessities. In order to answer these questions, large and representative samples are needed. Studies so far have been conducted with selective samples only (e.g., with employees who have been provided a smartphone by their employer; Derks et al. 2015).

\section{Study Aim}

Because of this, up until now it cannot be concluded a) how many employees engage in context-incongruent smartphone use, b) how much employees engage in contextincongruent smartphone use, c) whether employees are likely to engage in both of the two context-incongruent smartphone uses to the same extent or are likely to engage in one more than the other, and d) whether certain characteristics of the work context or the individual make it more likely that context-incongruent smartphone use occurs. We attempted to get insight into a) - c) by conducting an exploratory latent class analysis (LCA) of work-related smartphone use at home and private smartphone use at work to identify subgroups of context-incongruent smartphone users. We attempted to get insight into d) by describing differences in demographic, smartphone-related, and occupational health-related characteristics between the identified classes. LCA is a person-centered statistical technique that identifies unobserved subgroups of individuals, using a set of measured variables as input, in a data-driven manner. We chose for this approach as the LCA allows us to study both types of context-incongruent smartphone use simultaneously while treating them as separate constructs, and because we were agnostic toward the number and kind of classes we could expect. We chose not to perform any confirmatory tests as we had no way of predicting the results of the LCA a priori. As a consequence, we want to emphasize that our intention was to merely characterize these data-driven classes along further variables, not to treat them as antecedents and outcomes.

We planned to characterize our identified classes along three sets of variables. As a first and general indication of potential differences, we wanted to see whether the classes differ in terms of demographics. Second, we probed several details and cognitions 
surrounding the smartphone. For example, we wanted to see whether the classes differed in the likelihood that they were provided a smartphone by their employer, how much personal importance they attached to their work-related and private smartphone interactions, and to what extent they are afraid of missing out on relevant and timely developments in their work and private life. From the perspective of boundary theory, these variables were meant to give us insight into the personal preferences and work environment with regard to the boundary-crossing behavior itself (i.e., contextincongruent smartphone use). Third, we chose to include several occupational healthrelated variables. We chose for variables that were empirically linked to smartphone use in previous work (segmentation preferences, psychological detachment, work-home conflict) as well as the two most studied work characteristics (job demands \& job control). From the perspective of boundary theory, this set of variables was meant to give us insight into the (perceptions of) context in which the boundary-crossing behavior takes place, as well as potential consequences of the boundary crossing.

While the smartphone can be (and often is being) used for context-congruent matters (i.e., work-related smartphone use at work and private smartphone use at home), here we are interested in boundary-crossing behavior. As a consequence, context-congruent smartphone use is beyond the scope of this study.

\section{Method}

\section{Participants and Procedure}

The sample came from a panel of participants of a nation-wide survey (Netherlands Working Conditions Survey (NWCS), year 2014) conducted by the Netherlands Organisation for Applied Scientific Research (TNO; Hooftman et al. 2015). This original survey was sent to 143,989 Dutch employees, of which 41,657 participated (response rate $28.9 \%$ ). Of these participants, 9855 were working $32 \mathrm{~h}$ or more per week (2014) and gave their permission to be contacted for follow-up surveys. We chose to limit our sample to employees working $32 \mathrm{~h}$ or more because contextincongruent smartphone use may qualitatively differ between people that work four to five days per week and those working only two to three days per week, which was not the focus of this study. In the spring of 2016 we sent this sub-sample a survey invitation via e-mail. In the invited sub-sample, 2116 filled in our questionnaire (response rate $21.5 \%$ ). Of this sample, we excluded 572 participants who i) now worked less than $32 \mathrm{~h}$ per week (123 participants) or ii) indicated that they did not own a smartphone at the time of filling in the survey (106 participants), or (iii) who did not fill in the questions about smartphone use (343 participants). This left us with a final sample of 1544 (75.1\% male) individuals $\left(\mathrm{M}_{\mathrm{age}}=47.23, S D_{\mathrm{age}}=10.64\right.$; $47.8 \%$ held at least a Bachelor's degree; $M_{\text {weekly work hours }}=39.73, S D_{\text {weekly work hours }}=$ 5.70). A more complete description of the overall sample can be found in the supplementary online materials. Compared to the sub-sample of the original study that worked more than $32 \mathrm{~h}$ per week (which is representative of Dutch employees; $n=22,060$ ), our participants were about five years older on average $\left(\mathrm{G}_{\text {Hedges }}=0.44\right)$, slightly more likely to be male $(\mathrm{OR}=1.26)$, and slightly more likely to hold a bachelor's degree $(\mathrm{OR}=1.36)$. 


\section{Measures}

If not indicated otherwise, items were answered on a 5-point Likert scale ranging from 1 (strongly disagree) to 5 (strongly agree).

Demographics We asked participants to indicate their age, gender, highest completed education, current relationship status (married/in a relationship vs single), weekly working hours, occupation, most common workplace (at employer's site vs at home/at different places), and whether they are in charge of supervising any number of employees. We collected this demographic information in order to describe our sample as a whole as well as our identified smartphone user classes.

Work-Related Smartphone Use at Home \& Private Smartphone Use at Work We developed four items to measure context-incongruent smartphone use. We created new items because we wanted the items to be semantically mirrored for both context-incongruent uses as well as smartphone using and smartphone checking, which was not practically possible with items used in previous work (e.g., Derks et al. 2015). The distinction between checking and using behavior was made because two individuals who use the smartphone to the same extent, but differ in the degree to which they check for messages, exhibit different interaction patterns. Two items measured how often participants use (i.e., manipulating the smartphone beyond checking) their phone for work-related (private) matters at home (work) (i.e., "How often do you use your smartphone for work-related matters at home on an average working day?"; "How often do you use your smartphone for private matters at work on an average working day?'), and two items measured how often participants check (for messages/notifications without further action) their phone while at home (work) (i.e., "How often do you check your smartphone at home on an average working day?"; "How often do you check your smartphone at work on an average working day?'). The reference to a workrelated/private purpose was removed from the checking items because the smartphone is not always checked with a specific purpose in mind. An earlier study (Kobayashi and Boase 2012) has shown that people make systematic errors when self-reporting the exact frequency of their smartphone use. With the aim of minimizing this bias we decided to offer our participants broad answer options on a 6-point Likert scale (1 = "never", "once a day", "several times a day", "once an hour", "several times an hour", 6 = "every few minutes").

Fear of Missing out We adapted the three items used by Reinecke et al. (2016) measuring fear of missing out when using the internet less. Our items reflected fear of missing out when using the smartphone less for work-related matters at home and for private matters at work (e.g., "If I would use my smartphone less for work-related (private) matters while at home (work), I would fear missing out on important things."; $\alpha_{\mathrm{W}}=.83 \& \alpha_{\mathrm{NW}}=.81$ ). In order to test whether the items used for FOMO at home and at work measured separate constructs, we conducted a confirmatory factor analysis. This analysis indicated that a model loading work-related and private FOMO on separate factors had a better fit $(\chi 2(8)=534.08, p<.001, \mathrm{CFI}=.88, \mathrm{SRMR}=.07, \mathrm{RMSEA}=.21)$ than loading them onto the same factor $(\chi 2(9)=1525.13, p<.001, \mathrm{CFI}=.67, \mathrm{SRMR}=.12$, RMSEA =.33), meaning that the two constructs can be empirically distinguished from one another. 
Smartphone-Related Questions We asked participants with self-developed 1-item measures to what extent their job is dependent on daily usage of their smartphone ("For the optimal execution of my job, daily use of a smartphone is necessary.") and to what extent their work-related use at home and private use at work is of personal importance to them ("The work-related (private) things that I do on my smartphone at home (work) are very important to me).

Job Demands Three items were used from the Netherlands Working Conditions Survey (Hooftman et al. 2015). The items (e.g., "Do you need to work really fast?") were answered on a 4-point scale $(0=$ Never, $3=$ Always; $\alpha=.85)$.

Job Control Six items from the Netherlands Working Conditions Survey (Hooftman et al. 2015) were used. The items (e.g., "Can you decide yourself on your work pace?”) were answered on a 3 -point scale $(1=$ No, $2=$ Yes, sometimes, $3=$ Yes, regularly; $\alpha=.76)$.

Segmentation Preferences To measure work-home segmentation preferences, three items from Kreiner's (2006) 4-item scale were used (e.g., "I don't like to have to think about work while I'm at home."). One item was dropped due to difficulties mirroring it to reflect segmentation preferences at work. The items showed sufficient internal reliability $(\alpha=.76)$. We mirrored these three items to reflect home-work segmentation preferences $(\alpha=.80)$. We conducted a confirmatory factor analysis to see whether segmentation preferences at home and at work were separate concepts. This analysis indicated that a model loading work-home and home-work segmentation preferences on separate factors had a better fit $(\chi 2(8)=109.26, p<.001, \mathrm{CFI}=.97, \mathrm{SRMR}=.04$, RMSEA $=.09)$ than loading them onto the same factor $(\chi 2(9)=741.53, p<.001$, $\mathrm{CFI}=.78, \mathrm{SRMR}=.10, \mathrm{RMSEA}=.23)$, meaning that the two constructs can be empirically distinguished.

Psychological Detachment The four respective items from the Recovery Experience Questionnaire (Sonnentag and Fritz 2007) were used (e.g., "During my leisure time after work, I distance myself from work"; $\alpha=.88$ ).

Work-Home/Home-Work Interference To measure negative WHI (HWI), we used the 8 (4) respective items from the SWING questionnaire (Geurts et al. 2005). The items (e.g., "How often does it happen that your work schedule makes it difficult for you to fulfill your domestic obligations?"; "How often does it happen that your performance at work suffers due to problems with your partner/family/friends?") were answered on a 4-point Likert scale $(0=$ [almost $]$ never, $3=$ [almost $]$ always; $\left.\alpha_{\mathrm{WHI}}=.85 \& \alpha_{\mathrm{HWI}}=.76\right)$.

Data Analytic Procedure To identify subgroups of context-incongruent smartphone users in an exploratory and data-driven manner, we conducted a latent class analysis with the poLCA package (Linzer and Lewis 2011) in R (v3.4.2; R Core Team 2017). Indicators of the LCA were our four items measuring using the smartphone for workrelated matters at home (1) and private matters at work (2) and checking the smartphone at home (3) and at work (4). We conducted LCAs that specified 2-10 classes and repeated these computations 10 times for robustness. We accepted the latent class structure with the best model fit as indicated by the Bayesian Information 
Criterion (BIC), which has been shown to be the best-performing information criterion for LCAs in a Monte Carlo simulation study (Nylund et al. 2007). After extracting the identified latent classes, we assigned individuals to their most likely class and compared them on several demographic, smartphone-related, and occupational healthrelated characteristics.

\section{Results}

\section{Latent Class Analysis}

The LCA identified a four-class model as the best fitting model according to the BIC. Table 1 gives an overview over the amount of context-incongruent smartphone use reported in the identified classes. The first class consisted of $15.2 \%(n=235)$ of participants that reported the highest smartphone checking at home and at work and using both for work-related matters at home and for private matters at work ("highhigh" class; hereinafter referred to as hh class). The second class consisted of $15.8 \%$ $(n=244)$ of participants that reported medium amounts of checking at home and workrelated smartphone use at home and high amounts of checking at work and private smartphone use at work ("medium-high" class; hereinafter referred to as mh class). The third class consisted of $41.1 \%(n=635)$ of participants that reported medium amounts of checking at home and at work and using for work-related matters at home and private matters at work ("medium-medium" class; hereinafter referred to as mm class). The fourth class consisted of $27.8 \%(n=430)$ of participants that reported the lowest checking at home and at work and using for work-related matters at home and private matters at work ("low-low" class; hereinafter referred to as 11 class). Figure 1 depicts the answering patterns on the four items in each of the identified classes. A more complete description of the identified classes can be found in the supplementary online materials.

\section{Differences among Latent Classes}

The reported average frequency of each item was highest in the hh class, second-highest in the mh class, third-highest in the mm class (one exception was reported work-related

Table 1 Means (1-6) and standard deviations of indicators of both types of smartphone use in the four latent classes of context-incongruent smartphone users

\begin{tabular}{|c|c|c|c|c|}
\hline & \multicolumn{2}{|c|}{ Work-related smartphone use at home } & \multicolumn{2}{|c|}{ Private smartphone use at work } \\
\hline & Checking & Using & Checking & Using \\
\hline & $M(S D)$ & $M(S D)$ & $M(S D)$ & $M(S D)$ \\
\hline High-high & $4.90(0.47)$ & $3.71(1.36)$ & $4.85(0.65)$ & $3.54(1.06)$ \\
\hline Medium-high & $3.48(1.33)$ & $2.11(0.97)$ & $4.43(0.50)$ & $3.49(0.79)$ \\
\hline Medium-medium & $3.23(0.62)$ & $2.28(0.85)$ & $3.30(0.63)$ & $2.68(0.54)$ \\
\hline Low-low & $2.23(1.10)$ & $1.35(0.63)$ & $2.62(0.86)$ & $1.84(0.67)$ \\
\hline
\end{tabular}




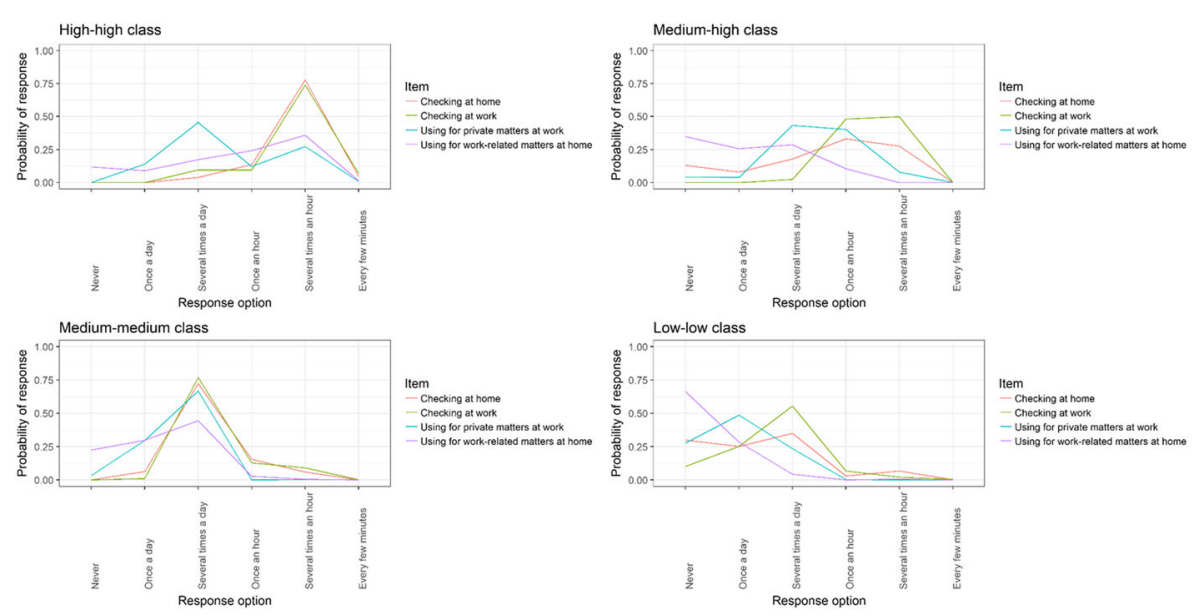

Fig. 1 Probabilities of response options on four items measuring smartphone use in the four identified classes

smartphone use at home, which was slightly higher in the mm class than in the mh class), and lowest in the 11 class. Because this observed gradient in the identified classes was somewhat mirrored in differences in demographic, smartphone-related, and occupational health-related characteristics, we chose to only present effect sizes for the differences between the hh class and the 11 class to be parsimonious (effects for comparisons between the rest of the classes are overall smaller in magnitude, which is consistent with the smaller differences in terms of the underlying reported smartphone use and can be found in the supplementary materials). We used odds ratios as an effect size for dichotomous variables and Hedges' $g$ for continuous variables $(0.2,0.5$, and 0.8 respectively were used as indicators of small, medium, and high values of Hedges' g). In case that the odds of the hh class were smaller than the odds of the 11 class, we reversed the order to ease interpretation of the odds ratios. Below, we give a verbal account of the differences between the hh class and the 11 class, as well as a description of how the $\mathrm{mh}$ class differs from its two adjacent classes (i.e., hh class and 11 class).

\section{Differences in Demographic Characteristics among Classes}

Table 2 summarizes the differences in demographic characteristics among the four classes. Compared to the 11 class, participants in the hh class were 5.65 years younger (medium difference) and worked $2.62 \mathrm{~h}$ more per week on average (small difference). They were 1.63 times as likely to be male and 2.60 times as likely to be married or in a relationship. Participants in the hh class were 1.65 times as likely to hold a university degree and were 1.63 times as likely to work in a white-collar occupation. Participants in the 11 class were 2.67 times as likely to work at their employer's site compared to at home or at varying places. Participants in the hh class were 1.76 times as likely to hold a leadership position.

\section{Differences in Smartphone-Related Characteristics among Classes}

Table 3 summarizes the differences in smartphone-related characteristics among the four latent classes. Participants in the hh class reported their job to be more dependent 
Table 2 Differences in demographic characteristics among the four latent classes

\begin{tabular}{|c|c|c|c|c|c|c|}
\hline & $\begin{array}{l}\text { High-high } \\
(n=235)\end{array}$ & $\begin{array}{l}\text { Medium-high } \\
(n=244)\end{array}$ & $\begin{array}{l}\text { Medium- } \\
\text { medium } \\
(n=635)\end{array}$ & $\begin{array}{l}\text { Low-low } \\
(n=430)\end{array}$ & Odds Ratio & $\mathrm{G}_{\text {Hedges }}$ \\
\hline & $M(S D)$ & $M(S D)$ & $M(S D)$ & $M(S D)$ & $(95 \% \mathrm{CI})$ & \\
\hline Age & $44.63(10.75)$ & $43.26(10.68)$ & $47.66(10.48)$ & $50.28(9.77)$ & & -0.56 \\
\hline \multirow[t]{2}{*}{ Working hours } & $41.17(6.82)$ & $39.47(5.32)$ & $40.09(5.69)$ & $38.55(4.97)$ & & 0.21 \\
\hline & $\%$ & $\%$ & $\%$ & $\%$ & & \\
\hline Gender & & & & & $1.63(1.12,2.38)$ & \\
\hline Male & $79.6 \%$ & $76.6 \%$ & $75.9 \%$ & $70.5 \%$ & & \\
\hline Female & $20.4 \%$ & $23.4 \%$ & $24.1 \%$ & $29.5 \%$ & & \\
\hline Relationship status & & & & & $2.60(1.61,4.19)$ & \\
\hline Married/relationship & $89.8 \%$ & $79.4 \%$ & $84.1 \%$ & $77.2 \%$ & & \\
\hline Single & $10.2 \%$ & $20.6 \%$ & $15.9 \%$ & $22.8 \%$ & & \\
\hline Education & & & & & $1.65(1.20,2.28)$ & \\
\hline University level & $53.8 \%$ & $59.8 \%$ & $45.2 \%$ & $41.4 \%$ & & \\
\hline Higher/lower secondary & $46.2 \%$ & $40.2 \%$ & $54.8 \%$ & $58.6 \%$ & & \\
\hline Occupation & & & & & $1.63(1.06,2.48)$ & \\
\hline White-collar & $65.0 \%$ & $68.6 \%$ & $62.4 \%$ & $53.3 \%$ & & \\
\hline Blue-collar & $35.0 \%$ & $31.4 \%$ & $37.6 \%$ & $46.7 \%$ & & \\
\hline Workplace & & & & & $2.67(1.88,3.79)$ & \\
\hline At employer's site & $60.0 \%$ & $65.6 \%$ & $69.1 \%$ & $80.0 \%$ & & \\
\hline At home/at varying places & $40.0 \%$ & $34.4 \%$ & $30.8 \%$ & $20.0 \%$ & & \\
\hline Leadership position & & & & & $1.76(1.27,2.46)$ & \\
\hline Yes & $42.6 \%$ & $33.6 \%$ & $38.0 \%$ & $29.6 \%$ & & \\
\hline No & $57.4 \%$ & $66.4 \%$ & $62.0 \%$ & $70.4 \%$ & & \\
\hline
\end{tabular}

Percentages do not need to add up to $100 \%$ due to rounding. Effect sizes relate to differences between the high-high and the low-low class

on daily usage of their smartphone (large difference) and attached more personal importance to their work-related smartphone use at home (medium difference) and to their private smartphone use at work (small difference). They reported higher fear of missing out at home (large difference) and at work (large difference). Compared to the 11 class, participants in the hh class were 4.34 times as likely to be provided a smartphone by their employer and were more likely to receive push notifications to the lock screen of their smartphone.

\section{Differences in Occupational Health-Related Characteristics among Classes}

Table 4 summarizes the differences in occupational health-related characteristics among the four latent classes. Participants in the hh class reported higher job demands and job control (small difference). Participants in the 11 class reported higher segmentation preferences at home (medium difference) and at work (medium difference). They also reported higher psychological detachment (medium difference). Participants in the hh class reported higher work-home interference (small difference). However, it must be 


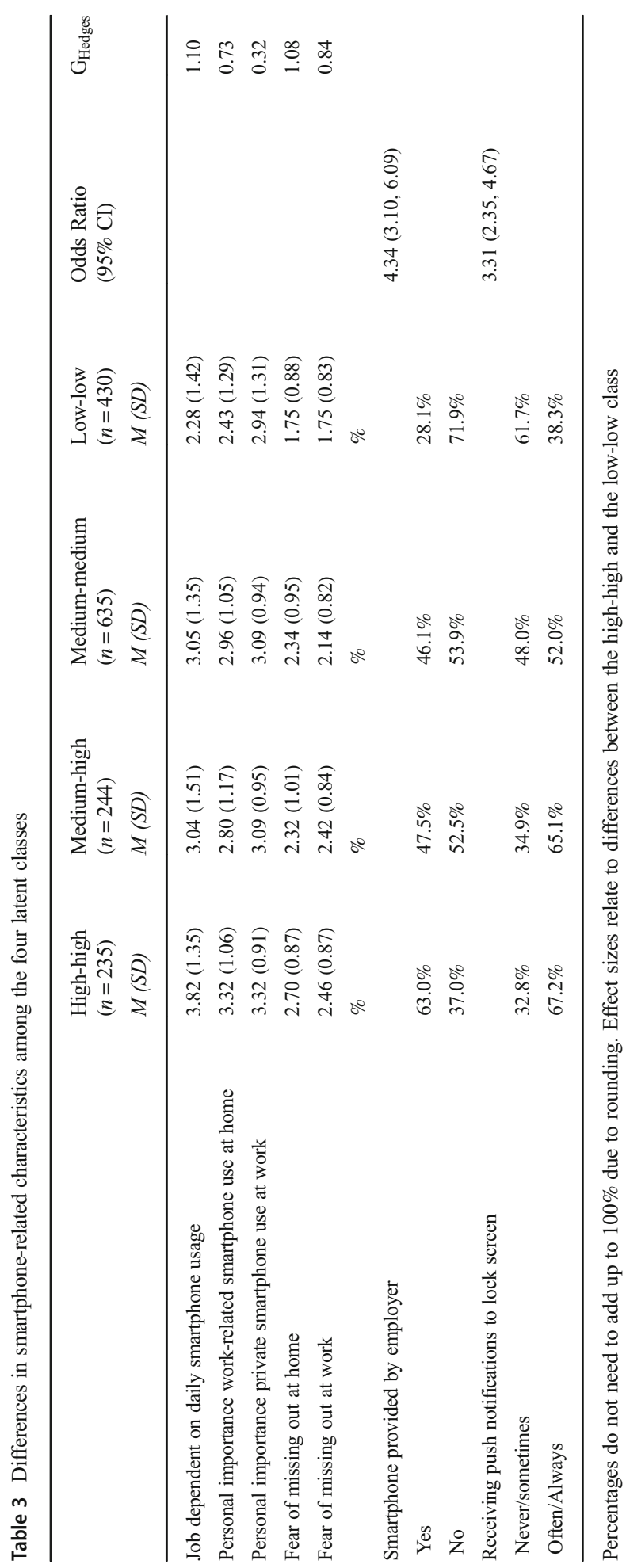


Table 4 Differences in occupational health-related characteristics among the four latent classes

\begin{tabular}{llllll}
\hline & $\begin{array}{l}\text { High-high } \\
(n=235) \\
M(S D)\end{array}$ & $\begin{array}{l}\text { Medium-high } \\
(n=244) \\
M(S D)\end{array}$ & $\begin{array}{l}\text { Medium-medium } \\
(n=635)\end{array}$ & $\begin{array}{l}\text { Low-low } \\
(n=430)\end{array}$ & $\mathrm{G}_{\text {Hedges }}$ \\
& $1.59(0.62)$ & $1.37(0.59)$ & $1.49(0.59)$ & $1.47(0.61)$ & 0.20 \\
\hline Job demands & $2.62(0.43)$ & $2.58(0.41)$ & $2.54(0.43)$ & $2.44(0.45)$ & 0.41 \\
Job control & $2.80(0.95)$ & $3.90(0.91)$ & $3.07(0.83)$ & $3.37(0.85)$ & -0.64 \\
Segmentation preferences at home & $2.90(0.84)$ & $3.02(0.81)$ & $3.21(0.77)$ & $3.41(0.78)$ & -0.64 \\
Segmentation preferences at work & $2.56(0.83)$ & $2.91(0.86)$ & $2.81(0.84)$ & $3.10(0.82)$ & -0.65 \\
Psychological detachment & $0.61(0.48)$ & $0.54(0.48)$ & $0.57(0.46)$ & $0.51(0.44)$ & 0.22 \\
Work-home interference & $0.24(0.40)$ & $0.23(0.33)$ & $0.21(0.30)$ & $0.21(0.31)$ & 0.09 \\
Home-work interference & & & & &
\end{tabular}

Percentages do not need to add up to $100 \%$ due to rounding. Effect sizes relate to differences between the high-high and the low-low class

noted that reported work-home interference was quite low in both classes. The differences regarding home-work interference were very small.

\section{The Mh Class Compared to the Hh Class and Mm Class}

The mh class is standing out as the only observed class in our sample that reported different relative amounts of context-incongruent smartphone use. This class reported comparable amounts of work-related smartphone use at home to the mm class and comparable amounts of private smartphone use at work to the hh class. In terms of demographics, notably the mh class was about as old as the hh class (very small difference) and younger than the mm class (medium difference). Participants in the $\mathrm{mh}$ class were 1.28 times as likely to have a university degree compared to the hh class, and 1.81 times as likely to have a university degree compared to the mm class.

Regarding the measured smartphone-related characteristics, the mh class was closer to the hh class (very small difference) compared to the mm class (small-to-medium difference) on fear of missing out at work, but more similar to the mm class (no difference) compared to the hh class (small difference) on the personal importance attached to private smartphone use at work. The mh class was closer to the mm class (very small-to-small differences) compared to the hh class (medium differences) on items reflecting cognitions toward work-related smartphone use at home (i.e., job dependence on daily smartphone usage, personal importance attached to work-related smartphone use at home, fear of missing out at home). Participants in the hh class were 1.87 times as likely to have been provided a smartphone by their employer compared to participans in the mh class, while participants in the mh class were 1.06 times as likely to have been provided a smartphone by their employer compared to participants in the mm class.

The mh class reported slightly lower job demands compared to both adjacent classes (small differences) and did not differ much from either with regard to reported job control (very small differences). However, the mh class reported lower segmentation preferences at home than both classes (large differences), and did not differ much compared to either class with regard to segmentation preferences at work (small 
differences). Finally, the mh class reported higher psychological detachment than the hh class (medium difference), but did not differ much from the mm class (very small difference). The mh class did not differ much from either class in terms of reported work-home and home-work interference (very small differences).

\section{Discussion}

The smartphone can be used for two context-incongruent purposes (i.e., work-related use at home and private use at work). In this study, we used LCA to identify distinct subgroups of context-incongruent smartphone users and to obtain insight into individual and environmental characteristics of these subgroups. By doing this, we attempted to answer (a) how many employees engage in context-incongruent smartphone use, (b) how much they engage in context-incongruent smartphone use, (c) whether employees that frequently use the smartphone for work-related matters at home are the same that frequently use the smartphone for private matters at work, and (d) whether we can distinguish between different groups of smartphone users in terms of individual and work-contextual characteristics.

Four latent classes were identified in this large and heterogeneous sample. These classes followed a gradient on our four items measuring smartphone use, with the hh class reporting frequent checking at home and at work and using of the smartphone for work-related matters at home and private matters at work, the 11 class barely reporting any checking and using in both contexts, and two classes in between. With regard to the questions posed in the introduction, this shows that (a) in this large and heterogeneous sample most employees use their smartphone for context-incongruent purposes, albeit (b) to strongly varying degrees ('once a day' to 'several times an hour'). Of note, (c) no class was identified that used the smartphone frequently in one context but rarely in the other context. This may be considered surprising given the very different connotations associated with both uses (work at home versus leisure at work) and may point to a more general decision each individual makes whether and to what extent to make use of the smartphone, irrelevant of use and context. Interestingly, participants reported to check and use the smartphone for private matters at work slightly more often compared to checking and using it for work-related matters at home. This finding further reinforces the need to study the distractive potential of private smartphone use at work, but also whether it may act as a recovery opportunity if used in the correct way.

Several interesting insights were obtained from (d) the comparison of the hh class and the 11 class. Our results indicate that younger employees use their smartphone more, and that this holds true both for work-related matters at home and private matters at work. To our surprise, participants in the hh class were much more likely to be married or in a relationship than participants in the 11 class. This could imply that employees mostly use their smartphone during work to communicate with their spouse and that they "re-pay" this flexibility during working hours with using the smartphone for workrelated matters in their leisure time (possibly due to their perceptions of fairness; Adams 1965). The analysis also showed that employees in the hh class were much less likely to work from their employer's site. This supports the intuitive notion that the smartphone as a mobile device is being used to work from anywhere, and it seems that the same employees also choose to use it to interact with their private life during work. 
Several large differences were observed regarding smartphone-related characteristics between the hh class and the 11 class. Participants in the hh class were more than four times as likely to be provided a smartphone by their employer, they rated their job as a lot more dependent on using their smartphone, they attached more importance both to their work-related and private smartphone interactions, and were more afraid of missing out on relevant short-term developments both in their work and private context. They were also much more likely to have push notifications installed on their smartphone. Overall, these findings indicate that frequent context-incongruent smartphone users rely more heavily on the smartphone both from the perspective of their job and from their personal needs.

Finally, our findings imply several interesting insights regarding contextincongruent smartphone use from an occupational health perspective. Participants in the hh class reported only slightly higher job demands and job control. Furthermore, participants in the hh class reported a lower preference to separate their work and private life both while at home and while at work. This extends an earlier study that found a link between segmentation preferences at home and higher work-related technology use at home (Park et al. 2011). The hh class also reported lower psychological detachment, which extends previous work that found a negative association between work-related smartphone use at home and psychological detachment (Derks et al. 2014b). As psychological detachment is crucial for off-job recovery to be effective (e.g., Sonnentag and Bayer 2005), our finding adds to the growing literature that indicates that using the smartphone for work-related matters may impede off-job recovery. Lastly, the differences regarding WHI between the hh class and the 11 class were small at best. This finding is at odds with two diary studies that found a positive relationship between work-related smartphone use at home and WHI (Derks and Bakker 2014; Derks et al. 2015), but are in line with another diary study that did not find this relationship (Derks et al. 2014a). However, it must be noted that our sample reported a lot less WHI compared to the convenience samples used in these diary studies.

While the identification of classes (and assignment of participants to classes) depended upon the report of both types of smartphone use, the mh class offers some unique insights. That is because this class is very similar to the hh class with regard to private smartphone use at work, and very similar to the mm class with regard to workrelated smartphone use at home. As such, comparing the mh class to the hh class and the mm class allowed us to disentangle which type of smartphone use (if only one) was related to the differences on the measured variables. Given that the mh class was relatively young and highly educated (similar to the hh class), this could mean that age and education relate stronger to the extent to which one uses the smartphone for private matters at work.

For the most part, the mh class was similar to the hh class regarding cognitions surrounding private smartphone use at work, and similar to the mm class regarding cognitions surrounding work-related smartphone use at home. An exception was that participants in the mh class reported less personal importance attached to their private smartphone use at work (similar to mm class). In that case, the mh class could have had some other motivation to use the smartphone a lot for private matters at work (such as high fear of missing out). It also appears that being provided a smartphone by one's employer relates much more to work-related smartphone use at home. This could imply 
that having a 'work' smartphone increases the perceived necessity or wish to use it for work-related matters at home without changing the employee's intentions to make use of the smartphone to take care of private matters while at work. Somewhat surprisingly, the mh class reported much higher segmentation preferences at home compared to the $\mathrm{mm}$ class. According to boundary theory, segmentation preferences should relate directly to the amount of boundary-crossing behavior, and hence here higher segmentation preferences at home should be reflected in less frequently reported work-related smartphone use at home. One possible explanation is that, because participants in the mh class use their smartphone a lot for private matters at work, they feel like compensating by also using it more for work-related matters at home. But it is also possible that some other combination of variables, measured or unmeasured, is the reason why this class reports high work-related smartphone use at home relative to the reported segmentation preferences. The mh class was much closer to the mm class in terms of reported psychological detachment, which suggests that psychological detachment does not relate strongly to private smartphone use at work. Overall, the mh class is much more similar to the hh class and mm class than to the 11 class on the measured variables. Differences from either of the two adjacent classes highlight which variables relate stronger to either context-incongruent smartphone use.

Taken together, our exploratory results provide some support for the predictions made by boundary theory. At least on a correlational level, measured personal preferences as well as environmental pressures differed between the identified classes, and hence related to the amount of boundary-crossing behavior reported. The classes also differed with regard to one of the two commonly studied outcomes of boundary crossing. Further research is needed to determine whether these results can be replicated with temporal separation between the theorized predictors, behavior, and outcomes.

\section{Limitations and Future Directions}

This study had several limitations that need to be emphasized when considering our findings. We have already discussed the problems associated with self-reporting smartphone use in the method section. However, given that collecting log data is not feasible when one aims to recruit such a large sample, we believe that making use of self-reports was an adequate choice in light of our research aims. Nonetheless, future studies should consider the use of $\log$ data to accurately capture participants' smartphone use frequency. Additionally, two of the items that served as input to our latent class analysis (checking the smartphone at home and at work) do not reflect context-incongruent smartphone use in $100 \%$ of the cases. Hence, our four identified latent classes are not based solely on the amount of context-incongruent smartphone use reported but also on an indicator of how often the participants pick up their smartphone at home and at work in general. We have already argued for the addition of these questions in the method section. To see whether this affected our results in a major way, we repeated our analyses only with the two context-incongruent smartphone use items as input to the LCA. This analysis resulted in two classes (hh and 11) that differed on our remaining measures in the same directions to our main analysis, albeit somewhat smaller in magnitude. Because all of our measures were self- 
reported, it is possible that common method bias (Podsakoff et al. 2003) had an influence on the effect sizes obtained in our study. However, Spector (2006) showed convincingly that mono-method correlations are not necessarily higher than multimethod correlations. Finally, our sample was not fully representative of the Dutch working population. As our sample only included employees who worked (almost) fulltime, it remains unknown if this study's findings can be generalized to workers employed for different numbers of hours. Furthermore, our sample's demographics differed slightly from those of the population of Dutch employees. Regarding this latter issue, we argue that the age difference is most relevant for our main measure of smartphone use. Given that younger people are more likely to be intensive smartphone users, this should mean that the size of our hh class is slightly conservative.

Despite these limitations, we believe that the results of our study contribute to understanding work-related smartphone use at home and private smartphone use at work. We showed that a majority of employees engages in both behaviors and identified four classes of context-incongruent smartphone users. Our results also indicated that these classes differ in terms of demographics, cognitions surrounding the smartphone and its uses, as well as cognitions and perceptions surrounding one's work. Ultimately, in combination with additional research, this knowledge may help organizations to develop policies that help their employees to maximize their workrelated and non-work-related well-being while maintaining high productivity.

Ideally, the results from this exploratory study should be used to formulate hypotheses that should be tested using pre-registered studies that objectively quantify smartphone use through logging data from the smartphone directly. For example, within the context of boundary theory, it would be interesting to test why employees engage in context-incongruent smartphone use (e.g., because they are afraid of missing out on important developments in the incongruent domain, or because they have been provided a smartphone by their employer, ...) and whether this makes a difference in terms of potential negative outcomes.

\section{Compliance with Ethical Standards}

Conflict of Interest On behalf of all authors, the corresponding author states that there is no conflict of interest.

Open Access This article is distributed under the terms of the Creative Commons Attribution 4.0 International License (http://creativecommons.org/licenses/by/4.0/), which permits unrestricted use, distribution, and reproduction in any medium, provided you give appropriate credit to the original author(s) and the source, provide a link to the Creative Commons license, and indicate if changes were made.

\section{References}

Adams, J. S. (1965). Inequity in social exchange. In L. Berkowitz (Ed.), Advances in experimental social psychology (pp. 267-299). New York: Academic press.

APA Center for Organizational Excellence (2013). Communication technology: Implications for work and well-being. Retrieved from http://www.apaexcellence.org/assets/general/2013-work-and-communicationtechnology-survey-final.pdf. Accessed 2 Feb 2013. 
Ashforth, B. E., Kreiner, G. E., \& Fugate, M. (2000). All in a Day's work: Boundaries and micro role transitions. The Academy of Management Review, 25, 472-491. https://doi.org/10.5465/AMR.2000.3363315.

Demerouti, E., Derks, D., ten Brummelhuis, L. L., \& Bakker, A. B. (2014). New ways of working: Impact on working conditions, work-family balance, and well-being. In C. Korunka \& P. Hoonakker (Eds.), The impact of ICT on quality of working life. Dordrecht: Springer Netherlands.

Derks, D., \& Bakker, A. B. (2014). Smartphone use, work-home interference, and burnout: A diary study on the role of recovery. Applied Psychology, 63, 411-440. https://doi.org/10.1111/j.1464-0597.2012.00530.x.

Derks, D., ten Brummelhuis, L. L., Zecic, D., \& Bakker, A. B. (2014a). Switching on and off...: Does smartphone use obstruct the possibility to engage in recovery activities? European Journal of Work and Organizational Psychology, 23, 80-90. https://doi.org/10.1080/1359432X.2012.711013.

Derks, D., van Mierlo, H., \& Schmitz, E. B. (2014b). A diary study on work-related smartphone use, psychological detachment and exhaustion: Examining the role of the perceived segmentation norm. Journal of Occupational Health Psychology, 19, 74-84. https://doi.org/10.1037/a0035076.

Derks, D., van Duin, D., Tims, M., \& Bakker, A. B. (2015). Smartphone use and work-home interference: The moderating role of social norms and employee work engagement. Journal of Occupational and Organizational Psychology, 88, 155-177. https://doi.org/10.1111/joop.12083.

Geurts, S. A. E., Taris, T. W., Kompier, M. A., Dikkers, J. S., van Hooff, M. L., \& Kinnunen, U. M. (2005). Work-home interaction from a work psychological perspective: Development and validation of a new questionnaire, the SWING. Work and Stress, 19, 319-339. https://doi.org/10.1080/02678370500410208.

Hooftman, W.E., Mars, G.M.J., Janssen, B., de Vroome, E.M.M., \& van den Bossche, S.N.J. (2015). Nationale Enquête Arbeidsomstandigheden 2014: Methodologie en globale resultaten. Retrieved from https://www.cbs.nl/NR/rdonlyres/0B49D1CA-D991-45A4-8012-F4DF5CA7D9ED/0/nea2014 methodologischrapport.pdf. Accessed 2 Feb 2018.

Kobayashi, T., \& Boase, J. (2012). No such effect? The implications of measurement error in self-report measures of Mobile communication use. Communication Methods and Measures, 6, 126-143. https://doi. org/10.1080/19312458.2012.679243.

Kreiner, G. E. (2006). Consequences of work-home segmentation or integration: A person-environment fit approach. Journal of Organizational Behavior, 27, 485-507. https://doi.org/10.1002/job.386.

Linzer, D. A., \& Lewis, J. B. (2011). poLCA: An R package for polytomous variable latent class analysis. Journal of Statistical Software, 42, 1-29. https://doi.org/10.18637/jss.v042.i10.

Nylund, K. L., Asparouhov, T., \& Muthén, B. O. (2007). Deciding on the number of classes in latent class analysis and growth mixture modeling: A Monte Carlo simulation study. Structural Equation Modeling, 14, 535-569. https://doi.org/10.1080/10705510701575396.

Park, Y. A., Fritz, C., \& Lex, S. M. (2011). Relationships between work-home segmentation and psychological detachment from work: The role of communication technology use at home. Journal of Occupational Health Psychology, 16, 457-467. https://doi.org/10.1037/a0023594.

Podsakoff, P. M., MacKenzie, S. B., Lee, J. Y., \& Podsakoff, N. (2003). Common method biases in behavioral research: A critical review of the literature and recommended remedies. Journal of Applied Psychology, 88, 879-903. https://doi.org/10.1037/0021-9010.88.5.879.

R Core Team. (2017). R: A language and environment for statistical computing. Vienna: R Foundation for Statistical Computing URL http://www.R-project.org/.

Reinecke, L., Aufenanger, S., Beutel, M. E., Dreier, M., Quiring, O., Stark, B., Wölfling, K., \& Müller, K. W. (2016). Digital stress over the life span: The effects of communication load and internet multitasking on perceived stress and psychological health impairments in a German probability sample. Media Psychology, 0, 1-26. https://doi.org/10.1080/15213269.2015.1121832.

Schlachter, S., McDowall, A., Cropley, M., \& Inceoglu, I. (2017). Voluntary work-related technology use during non-work time: A narrative synthesis of empirical research and research agenda. International Journal of Management Reviews, 20, 825-846. https://doi.org/10.1111/ijmr.12165.

Sonnentag, S., \& Bayer, U. V. (2005). Switching off mentally: Predictors and consequences of psychological detachment from work during off-job time. Journal of Occupational Health Psychology, 10, 393-414. https://doi.org/10.1037//1076-8998.10.4.393.

Sonnentag, S., \& Fritz, C. (2007). The recovery experience questionnaire: Development and validation of a measure for assessing recuperation and unwinding from work. Journal of Occupational Health Psychology, 12, 204-221. https://doi.org/10.1037/1076-8998.12.3.204.

Spector, P. E. (2006). Method variance in organizational research: Truth or urban legend? Organizational Research Methods, 9, 221-232. https://doi.org/10.1177/1094428105284955.

Voorveld, H. A. M., \& van der Groot, M. (2013). Age differences in media multitasking: A diary study. Journal of Broadcasting \& Electronic Media, 57, 392-408. https://doi.org/10.1080/08838151.2013.816709. 
Publisher's Note Springer Nature remains neutral with regard to jurisdictional claims in published maps and institutional affiliations.

\section{Affiliations}

Jonas Dora ${ }^{1}$ • Madelon L. M. van Hooff ${ }^{1}$ • Sabine A. E. Geurts ${ }^{1}$ • Wendela E. Hooftman $^{1,2} \cdot$ Michiel A. J. Kompier ${ }^{1}$

Jonas Dora

j.dora@psych.ru.nl

1 Behavioural Science Institute, Radboud University, P.O. Box 9104, 6500 HE Nijmegen, The Netherlands

2 TNO, Leiden, The Netherlands 